\title{
The Assessment of Finishing Properties on the Mass per Unit Area, Pilling, Bursting Strength, and Wicking Behavior of Polyester Weft-Knitted Jersey Fabric
}

\author{
Tufail Hassan ${ }^{1}$, Muhammad Qamar Khan ${ }^{1, *}$, Abdul Salam ${ }^{1}$, Nafees Hassan ${ }^{1}$, Ali Raza ${ }^{1}$, \\ Nabi Bukhsh ${ }^{1}$, Zafar Javed ${ }^{2}$ and Ick Soo Kim ${ }^{3, *}$ \\ 1 Department of Textile \& Clothing, Faculty of Engineering \& Technology, National Textile University \\ Karachi Campus, Karachi 74900, Pakistan; tufailhassan12@gmail.com (T.H.); ab.salam8929@gmail.com (A.S.); \\ nafeeskhattak202@gmail.com (N.H.); aliraza@ntu.edu.pk (A.R.); nabibux29@gmail.com (N.B.) \\ 2 Department of Design, Faculty of Humanities and Social Sciences, National Textile University, \\ Faisalabad 37610, Pakistan; zafar@ntu.edu.pk \\ 3 Nanofusion and Technology Research Lab, Faculty of Textile Sciences, Shinshu University, Nagano 390-8621, \\ Japan; kim@shinshu-u.ac.jp \\ * Correspondence: drqamar@ntu.edu.pk (M.Q.K.); kim@shinshu-u.ac.jp (I.S.K.)
}

Received: 5 July 2020; Accepted: 22 July 2020; Published: 23 July 2020

\begin{abstract}
Finishes bring an alteration to the physical and comfort properties of the textiles. That's why various finishes are used to impart various functionalities to the fabric surface. However, it may also affect some properties. The purpose of this study is to investigate the effect of various finishes on pilling, mass per unit area, bursting strength, and wicking behavior of the polyester weft-knitted jersey fabric. Herein, 100\% spun polyester weft-knitted plain jersey fabric was exposed to different finish treatments to check their effect on the some physical and comfort properties of the fabric like mass per unit area, pilling behavior, bursting strength, and wicking properties of the weft-knitted jersey fabric. The fabric used was knit from $24 / 1,100 \%$ spun polyester yarn on the single knit circular knitting machine. The developed fabric was washed on Fong machine. Finishes are applied on fabric by "Monofort Stanter" machine. The resultant fabric was characterized by random tumble pilling tester, bursting strength tester, and wicking tester to analyze their pilling grade, bursting strength, and wicking behavior respectively. A significant increase has been found in wicking behavior, mass per unit area, and bursting strength of the fabric after finishing treatments. Moreover, the wicking finish shows the highest reduction in pilling grade from 3.5 to 2.5. Significant improvement has been observed in bursting strength by all finish's treatment. However, wicking finish treatment results in the highest increase in bursting strength of $4.2 \%$. Significant improvement has been observed in the vertical wicking speed of all treatment except silicon finish which significantly reduces vertical wicking rate. However, the wicking finish (Recipe E) shows the highest increase in wicking rate by 13.75 times as compared to grey fabric.
\end{abstract}

Keywords: weft knitted fabric; pilling; bursting strength; mass per unit area; wicking; polyester fabric; softeners; finishes

\section{Introduction}

Polyester fabric is widely used in our daily life for casual and sportswear applications. Various finishes are added to impart some specific functionality. Finishes also bring an alteration to the physical and comfort properties of the textiles. Therefore, it is important to check the effect of such finishes on some other properties like pilling, bursting strength, wicking behavior, and dimensional stability of the fabric. Pilling is an unwanted effect on the fabric surface caused by the protruding fibers of the 
yarn from which fabric is formed [1]. Protruding fibers come to the surface due to abrasion or wear. These fibers entangle and twisted together to form a small ball on the fabric surface known as a pill [2]. Pilling has two parts, anchor fibers, and pill. The fibers which hold the pill is called anchor fibers. More the strength of anchor fibers more will be difficult to wear of the pill [3]. Pilling greatly effects the appearance of the fabric [4]. Pilling tendency of synthetic fibers is higher than that of natural fiber due to its high strength which reduces wear off rate and pills appear on the surface easily [5]. In a blend of synthetic and natural fibers, synthetic fibers act as anchor fiber while natural fiber form pills [6]. Due to the high strength of synthetic fibers wear off rate reduces.

Except for fiber type, several factors affect the pilling. Alston et al. found that yarn manufacturing from the air jet technique is more pilling resistance than ring and rotor yarn. While ring yarn has more pilling resistance than rotor yarn. By increasing the twist of the yarn pilling reduces [7]. Shinvasan, et al. found that fiber denier has no significant effect on the abrasion and pilling resistance of the fabric [8]. Functional finishes alter the surface and mechanical properties [9]. Milind et al. studied the effect of resin finishes on the pilling behavior of knitted fabric. They used various constructions of the fabric and three types of finishes. Results reveal that finishes reduce the pilling tendency in most of the fabrics because resin finishes bind the protruding fibers with the surface [10]. Daiva Makucioniene has reported that the pilling of the fabric also depends on the structure of the fabric and linear density of the yarn. He found that $2 \times 2$ rib has more pilling resistance than $1 \times 1$ rib and interlock fabric. By decreasing fabric density and increasing yarn linear density pilling tendency increases [11].

Tausief et al. investigated that the $\mathrm{pH}$ was found to have no significant effect on pilling. They also check the effect of anti-pilling agents and found that by increasing the concentration of anti-pilling agents, pilling decreases [12]. Azhar-ul-Islam et al. reported that by increasing the ratio of polyester fiber in a blend, pilling tendency also increases. The singed fabric has more pilling resistance than un-singed fabric. By increasing, mass per unit area (GSM) of the fabric pilling propensity also increases [13]. Cyniak and Popin investigated the effect of polyester fiber ratio in blends on the pilling behavior of fabric. They found that increasing polyester fiber ratio in blends hairiness also increases which directly contributes to increasing pilling. Brand et al. found that in single jersey fabric fuzz formation and entanglement steps control the pilling while wear off did not play an important role [14]. Akhter et al. reported that combed yarn has less pilling tendency than carded yarn [15].

Bursting strength is the important parameter of knitted fabric and finishes can also affect it. Hussain et al. investigated the effect of softener finishes, resins, and curing time on the bursting strength of $100 \%$ cotton weft-knitted Lacoste pique fabric. Results revealed that by increasing resin concentration cause to deteriorate bursting strength of the fabric. However, by increasing softener concentration and curing time the bursting strength will also increase significantly [16]. Usha et al. studied the effect of knitted fabric type on bursting strength. They used single jersey fabric, interlock fabric, and single jersey pique fabric. The results show that the bursting strength of interlock fabric is higher than jersey and pique fabric. Moreover, the bursting strength of pique fabric is found to be higher than that of single jersey fabric [17] Oğuz et al. investigated the bursting strength of viloft/polyester blended weft-knitted single jersey and $1 \times 1$ rib fabric. They use different percentages of viloft/polyester 0/100,33/67, 50/50,67/33, and 100/0 for the manufacturing of 30/1 ring spinning yarn. Fabric produced was exposed to bursting strength test. Results reveal that by increasing polyester ratio in the blend the bursting strength will also be increased. Moreover, the bursting strength of the $1 \times 1 \mathrm{rib}$ is higher than the single jersey fabric [18]. Nida et al. conducted a study on the effect of inlay yarns amount on the bursting strength of compression stockings. Results reveal that by the incorporation of inlay yarn the bursting strength of the fabric shows significant improvement. $d$ [19]. Gong et al. investigated the effect of various softeners on bending properties of the knitted fabric. Results reveal that softeners reduce the bending properties of the interlock knitted fabric because of the reduction of inter yarn friction [20]. Khandaker et al. studied the effect of finishing on the bursting strength of the cotton-polyester blended (35\% polyester and $65 \%$ cotton) weft-knitted single jersey fabric. Results indicate that the bursting strength of heat setting fabric is lower than without heat setting fabric [21]. 
Wicking plays a key role in the comfort of any fabric. It has the ability of a fabric to evaporate moisture. Canen et al. investigated the comfort properties of compression garments. He studied the effect of elastane composition on the vertical wicking of single jersey plaited knitted fabric. The results show that by increasing in elastane composition percentage the vertical wicking of the fabric also increases. Moreover, they also investigated the effect of process tension on the vertical wicking of the fabric. The results reveal that low tension during the manufacturing process results in higher wicking as compared to higher tension [22]. Ikilem et al. conducted a study on the effect of Lycra percentage and some finishing treatments on vertical wicking behavior of seamless garments. They used conventional polyamide and meryl skinlife fibers for the manufacturing of fabric. The results expose that vertical wicking of the fabric significantly increases by increasing Lycra percentage. Moreover, applying antibacterial finishes causes reduction in vertical wicking rate because the yarn surface was all covered by finishing agents hence the water transport behavior also changed [23]. Yuliang et al. studied the incorporation of $\mathrm{ZnO}$ into Polyvinylidene fluoride (PVDF) and Polyacrylonitrile (PAN) nanofibers. The effect of hybrid PVDF/PAN/ZnO nanocomposite was investigated on antibacterial and wicking behavior. Significance reduction in vertical wicking of the nanocomposite has been observed by the incorporation of $\mathrm{ZnO}$ nanorods because it blocks pores available on the surface which affects vertical wicking speed [24].

Our local industries are facing a lot of challenges that significantly affect their production targets and business growth. One of the challenges is the lack of technical literature availability for a huge number of dyes and chemicals. Most of the time industries go for several trials to get some data for some specific finishes which needs a lot of time and huge financial resources. The current study was held to perform various trials of applying different finishes on $100 \%$ spun polyester weft-knitted jersey fabric and investigate their effect on the physical and comfort properties of the fabric. Herein, the effect of various finishes and washing treatments on the mass per unit area, pilling tendency, bursting strength, and wicking behavior of the $100 \%$ spun polyester weft-knitted plain jersey fabric has been investigated. Polyester fiber is widely used recently due to its low cost and ease of availability and its use is also increasing very rapidly. Industries are using different finishes on fabric to impart various functionality to achieve customer desire properties. Finishes provided by the manufacturer do not have technical literature which tells about the effect of such finishes on the properties of the fabric like pilling, bursting strength, areal density, and wicking behavior of the fabric. Hopefully, this study will help the users in a better selection of suitable finishes for imparting specific functions without compromising on other properties. This study will provide technical data to the industries about the few wet treatment recipes and their impact on various properties. Data of this study will save the time and financial resources of the industries.

\section{Materials and Methods}

Herein, polyester fibers were purchased from Ibrahim Fibers limited Pakistan, and the 24/1 $100 \%$ spun polyester yarn manufacturing was carried out by a ring spinning machine. A $100 \%$ spun polyester weft-knitted jersey fabric was knitted on an 18/22 diameter/gauge circular knitting single jersey machine. The specifications fibers, yarn, knitting machine, and fabric are shown in Table 1. The developed fabric is exposed to different wet processing finishing treatment as shown in Table 2.

Table 1. Fiber, yarn, fabric, and machine specifications.

\begin{tabular}{cc}
\hline Parameters Name & Specifications \\
\hline Composition & $100 \%$ Spun Polyester \\
Wpcm $^{1}$ & 12 \\
Cpcm $^{2}$ & 17 \\
Stitch Length & $0.295 \mathrm{~cm}$ \\
\hline
\end{tabular}


Table 1. Cont.

\begin{tabular}{cc}
\hline Parameters Name & Specifications \\
\hline Mass per unit area (Gsm) & 160 \\
Machine Name & Vanguard Supreme \\
Diameter & 18 \\
Gauge & 22 \\
Needles & 1248 \\
RPM $^{3}$ & 28 \\
Count Range & $24 / 1-40 / 1$ \\
Feeders & 18 \\
Linear Density & 1.3 denier \\
Fiber Length & 38 mm \\
Fiber Type & Polyester \\
Yarn Type & Ring-spun yarn \\
Material used & $100 \%$ Polyester \\
TPI & 14 \\
Count & 23.7 \\
CLSP & 5794 \\
Color & White
\end{tabular}

${ }^{1}$ Wpcm: Wales per centimeter; ${ }^{2} \mathrm{Cpcm}$ : Course per centimeter; ${ }^{3}$ RPM: Revolutions per minute; ${ }^{4}$ TPI: Twist per Inch; ${ }^{5}$ CLSP: Count lea strength product.

Table 2. Recipe Coding of Different Finishes.

\begin{tabular}{|c|c|c|c|}
\hline Fabric Treatment & Recipe Coding & Chemicals & Quantity $\left(g \cdot L^{-1}\right)$ \\
\hline $\begin{array}{c}\text { Gray Fabric } \\
\text { (Untreated Reference Sample) }\end{array}$ & A & N/A & N/A \\
\hline \multirow{2}{*}{$\begin{array}{c}\text { Simple Wash } \\
\text { (For Removing stains and oil marks }\end{array}$} & \multirow{2}{*}{ B } & Felosin RCN & 2 \\
\hline & & Diavan PCV & 2 \\
\hline \multirow{3}{*}{$\begin{array}{l}\text { Normal Finish } \\
\text { (For improving fabric hand } \\
\text { feel and softness) }\end{array}$} & \multirow{3}{*}{$\mathrm{C}$} & Acetic acid & 0.11 \\
\hline & & Polyavin PEN & 0.83 \\
\hline & & Founglube Softener & 0.5 \\
\hline \multirow{4}{*}{$\begin{array}{l}\text { Silicon Finish } \\
\text { (For improving the softness of the fabric) }\end{array}$} & \multirow{4}{*}{$\mathrm{D}$} & Acetic acid & 0.11 \\
\hline & & Polyavin PEN & 0.83 \\
\hline & & Magasil MT & 1 \\
\hline & & Cerofin SMT & 3.3 \\
\hline \multirow{3}{*}{$\begin{array}{c}\text { Wicking Finish } \\
\text { For Improving the wicking behavior of } \\
\text { the fabric }\end{array}$} & \multirow{3}{*}{$\mathrm{E}$} & Acetic acid & 0.11 \\
\hline & & Rucofin GSQ 200 & 1.5 \\
\hline & & Reapert SR & 1.5 \\
\hline
\end{tabular}

The developed fabric was cut into five pieces and marked on both sides for easy identification. One sample is considered as a reference while the remaining were exposed to various treatments as shown in Table 2. As wide practice of industries, fabric is exposed to washing treatment before dying to remove stains and oil spots in order to achieve better and uniform dying results. For this purpose, a recipe containing chemicals, e.g., Felosin RCN detergent and Diavan PCV, was prepared. Fabric was loaded to a Founge washing machine at $60{ }^{\circ} \mathrm{C}$ temperature for $20 \mathrm{~min}$. Later the sample was exposed to a Hydro machine for removing water. Macro-emulsion of silicon oil based Sarofin SMT (Chem-Tech international) is used as softeners for improving softness and hand feel of the fabric. A recipe of acetic acid (local), Polyavin PEN (CHT R.Beitlich GMBH Germany) and Megasil MT (Chem-Tech International) were applied on the fabric via a Santex machine (Swiss Textile Machinery, Zurich, Switzerland). Furthermore, in order to enhance the wicking behavior of the fabric a recipe of acetic acid (local), Rucofin GSQ 200 (Rudolf Chemie), and Reapert SR New (Bozzetto group) were 
applied on the fabric and then exposed to Monofort stanter at $120^{\circ} \mathrm{C}$ for $90 \mathrm{~s}$ to remove moisture. Moreover, normal finish contains recipe acetic acid (local), Foung lube and Polyavin PEN were applied in order to enhance hand feel of the developed sample.

During fabric manufacturing and finishing treatments Vanguard Supreme circular weft knitted jersey machine (Nova Knitting Machinery, Monroe, LA, USA), Monofort Fong's Stanter machine (Monforts Fong's Textile Machinery Co., Ltd., Mönchengladbach, Germany), Santex Machine, and Hydro Machine (Tongjiang Washing Machinery Factory, Taizhou, China) are used.

\subsection{Mass per Unit Area}

Mass per unit area is the key parameter of the knitted fabric for the negotiation between supplier and buyer. Therefore, it is very important to control it through various possible parameters. GSM cutter method was used to calculate the mass per unit area of the fabric. The first fabric was cut via GSM cutter of size $112 \mathrm{~mm}^{2}$ by following ASTM-D-3776 [25]. The weight of the cut fabric sample was done through digital weight balance. The mass per unit area (GSM) of fabric was calculated by Equation (1).

$$
\text { Mass per unit area }(\text { GSM })=\text { Sample Weight } \times 100
$$

\subsection{Pilling Test}

Pilling is an unwanted effect on the fabric surface caused by protruding fiber. It is very common in knitted fabrics due to fabric loose structure and the use of low twist yarn. Firstly, samples were cut in the size of $10.16 \times 10.16 \mathrm{~cm}^{2}$. Pilling test of all samples was performed on random tumble pilling tester by ASTM-D-3512 standard [26]. As per standard procedure, samples were kept in random tumble pilling tester for $30 \mathrm{~min}$. The pilling grade was evaluated by a standard replica having grades ranged from 5 to 1, which represents very good to poor, respectively.

\subsection{Bursting Test}

Bursting strength refers to the perpendicular force required to break or rupture the fabric. To investigate the bursting strength of the fabric, the sample was cut in the size of $112 \mathrm{~mm}^{2} \mathrm{by}$ using the standard method of ASTM-D-3786 [27]. Resulted samples were clamped over a rubber diaphragm and a diaphragm bursting strength tester was used for calculating the value of bursting strength. During testing cut samples were clamped over the rubber diaphragm and increasing fluid pressure was applied to the fabric until it burst. Noted the value of pressure against each sample.

\subsection{Vertical Wicking Test}

To investigate the wicking behavior, vertical wicking tester was used, and the sample was cut in the size of $165 \times 25 \mathrm{~mm}^{2}$ in both wales wise and course-wise direction. The resultant wale-wise samples were hanged vertically in wicking machine such that the $0.5 \mathrm{~mm}$ length of samples dips in the water and note the time. After $30 \mathrm{~min}$, samples were removed from the water and measure the total length to which water is traveled. The wicking speed of samples was measured by Equation (2). The same steps were repeated to investigate the wicking behavior of course-wise samples.

$$
\text { Wicking Speed }=(\text { Distance traveled by water in } \mathrm{mm} / \text { Total time in } \mathrm{min})
$$

\subsection{Yarn Testing}

Yarn quality was tested by UT-5 (S-400). The count was measured by wrapping the yarn over the reel and weight balance method. Moreover, the twist of the yarn was measured by the untwisting and re-twist method. 


\section{Results and Discussion}

\subsection{Effect of Finishes Treatment on the Mass per Unit Area}

During fabric manufacturing, the yarns are under high tension. Later, when the fabric is wet in a tensionless condition the relaxation occurs and the course-wise and wale-wise dimensions become shortened. Therefore, the capability of the shrinkage rate for various treatments would be a significant advantage. Moreover, the mass per unit area has a direct relation with fabric shrinkage. When the fabric is exposed to any type of wet processing treatment it undergoes some shrinkage. When fabric shrink it alters some other properties as well. Different wet processing treatments undergo different shrinkage. The results of this study reveal that the mass per unit area of the fabric is increased by all recipe treatments B, C, D, and E as shown in Figure 1 and Table 3. However, treatment D and E record the highest increase in mass per unit area. As single jersey fabric has unstable structure and all types of wet treatments undergo relaxation of internal stresses produced in the loops and fibers during the knitting process and yarn manufacturing process, respectively. Such relaxation of fibers and loops is responsible for shortening the fabric in both the wale-wise and course-wise dimensions. In other words, we can call it shrinkage of the fabric. That's why wet treatment of the fabric cause to increase in the mass per unit area. Due to shrinkage, the structure of the fabric becomes more compact and mass per unit area also increases [28].

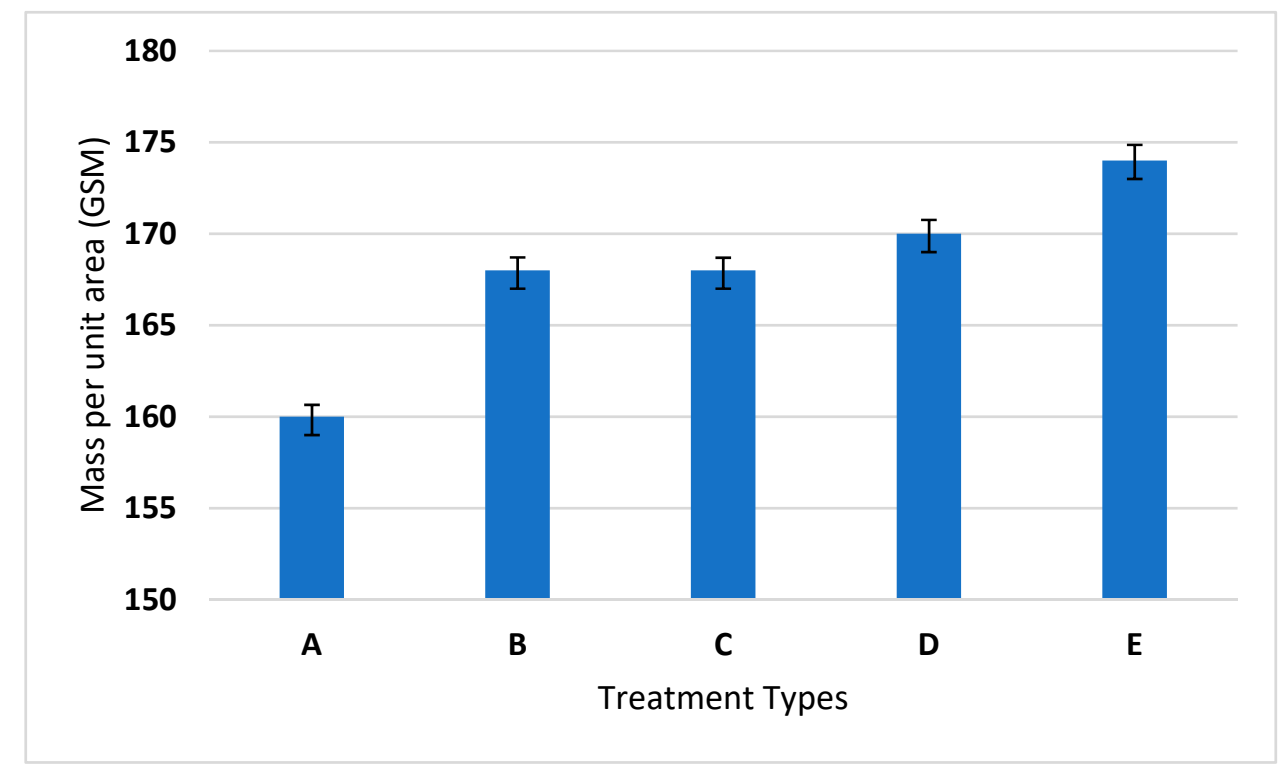

Figure 1. Effect of finishes on mas per unit area of the fabric (A) grey fabric, (B) simple wash, (C) normal finish, (D) silicon finish, and (E) wicking finish.

Table 3. Effect of finishes treatments on the mass per unit area (GSM).

\begin{tabular}{ccc}
\hline Treatment Type & Mass per Unit Area (GSM) & SD $( \pm)$ \\
\hline A & 160 & 0.65 \\
B & 168 & 0.71 \\
C & 168 & 0.69 \\
D & 170 & 0.76 \\
E & 174 & 0.86 \\
\hline
\end{tabular}

\subsection{Effect of Finishes Treatment on the Pilling of the Fabric}

The average pilling results of all the samples are exhibited in Table 4 and Figure 2. Treatment of fabric sample with recipe B cause deteriorate pilling grade from 3.5 to 2.5. Recipe C treated fabric 
shows half grade deterioration in the pilling grade from 3.5 to 3 . As treatment $\mathrm{D}$ is considered it causes to reduce the pilling resistance. In terms of grades, it shows reduction in pilling grade from 3.5 to 3. Lastly, the treatment of the fabric with recipe $\mathrm{E}$ also causes to reduce piling grade from 3.5 to 2.5 . To sum up, recipes B, C, D, and E showed a decrease in pilling resistance and pilling rating of the fabric. However, the results of the recipes $C$ and $D$ are comparatively appreciable which caused half grade deterioration in pilling as shown in Figure 2 and Table 4. On the other hand, recipes B and E caused the highest deterioration by one grade. The above results could be explained that during such treatments, the fraction between fabric and machines possibly caused to damage the surface fibers. Secondly, washing and finishing treatments caused increased wales and course density hence, yarns in the fabric come closer to each other. It causes to increase more protruding fiber per unit areas than untreated fibers, hence more pills appear per unit area [13]. Moreover, it is due to the ability of these softeners and finishes to reduce fiber to fiber friction by increasing individual fiber lubrication, thus, facilitating the fibers coming out of the main body of the fabric to form pills [13].

Table 4. Effect of finishes treatments on pilling grade.

\begin{tabular}{ccc}
\hline Treatment Type & Pilling Grade (-) & SD ( \pm ) \\
\hline A & 3.5 & 0.29 \\
B & 2.5 & 0.17 \\
C & 3 & 0.34 \\
D & 3 & 0.27 \\
E & 2.5 & 0.21 \\
\hline
\end{tabular}

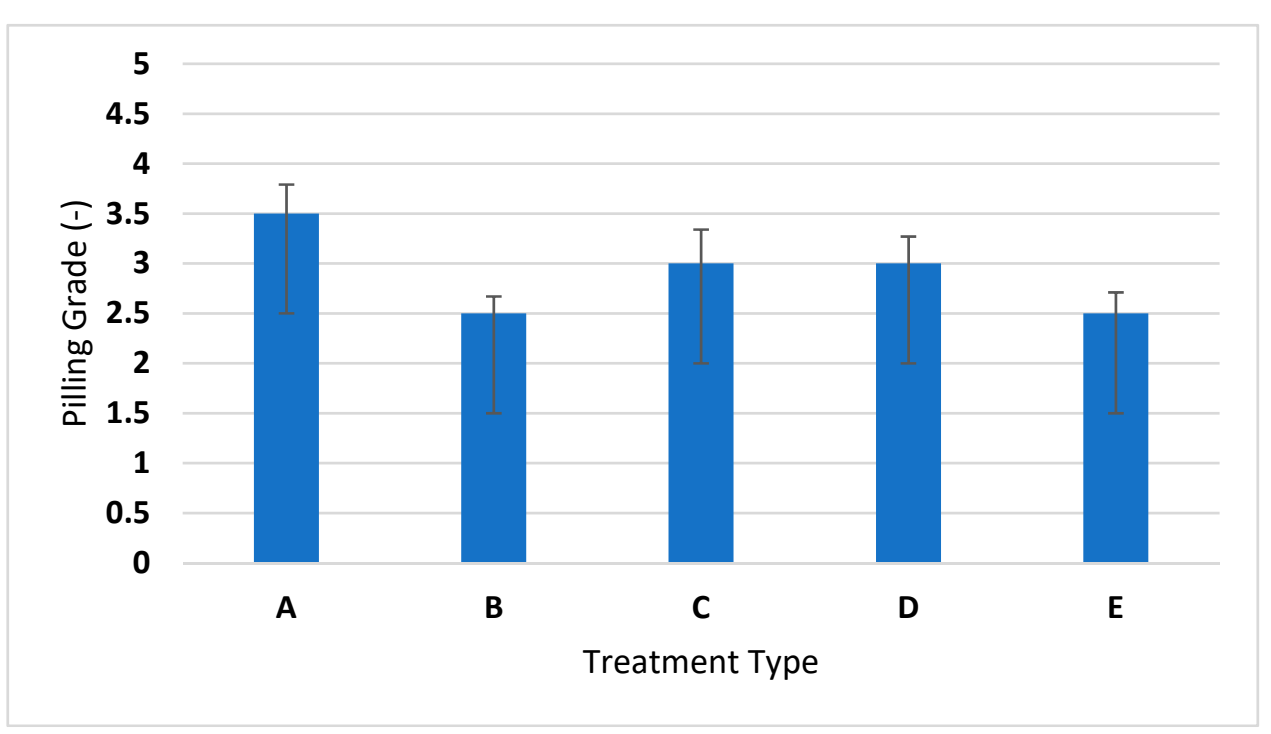

Figure 2. Pilling grades for different treatments (A) grey fabric, (B) simple wash, (C) normal finish, (D) silicon finish, and (E) wicking finish.

\subsection{Effect of Finishes Treatments on Bursting Strength}

Washing of fabric with recipe B cause to increase the bursting strength from 1.16 to 1.17 MPa. To improve the handfeel, of the fabric recipe, $\mathrm{C}$ was applied on the fabric and it has found that it causes to increase the bursting strength from 1.16 to $1.18 \mathrm{MPa}$.

To improve the hand-feel of the fabric, recipe D was applied to the fabric. The results reveal that the bursting strength increases from 1.16 to $1.19 \mathrm{MPa}$. To improve the wicking behavior of the fabric, recipe $\mathrm{E}$ was applied to the fabric. The results show an increase in bursting strength from 1.16 to $1.31 \mathrm{MPa}$ as shown in Table 5. So, overall treatments B, C, D, and E showed $1.2 \%, 1.8 \%, 2.4 \%$, and $4.2 \%$ increases in bursting strength, respectively. However, treatments $\mathrm{D}$ and $\mathrm{E}$ show more increase in bursting strength as compared to B and C as shown in Figures 3 and 4. Wet processing caused 
shrinkage and an increase in mass per unit area which has a direct relation to an increase in stitch density of the knitted fabric. Higher stitch density fabric required higher pressure to burst knitted fabric because a greater number of loops are prone to open and straightened before bursting [29]. Stiffer and brittle yarns can be easily broken then soft yarns and finishing treatments significantly reduce the stiffness of the yarns which leads to higher bursting strength of the fabric [30]. Moreover, softening treatment increased the smoothness of the yarn due to which yarn became more slippery when exposed to some force or pressure which caused an increase in bursting strength [16].

Table 5. Effect of finishes treatments on bursting strength (MPa).

\begin{tabular}{ccc}
\hline Treatment Type & Bursting Strength (MPa) & SD ( \pm ) \\
\hline A & 1.16 & 0.020 \\
B & 1.17 & 0.022 \\
C & 1.18 & 0.021 \\
D & 1.19 & 0.024 \\
E & 1.21 & 0.027 \\
\hline
\end{tabular}
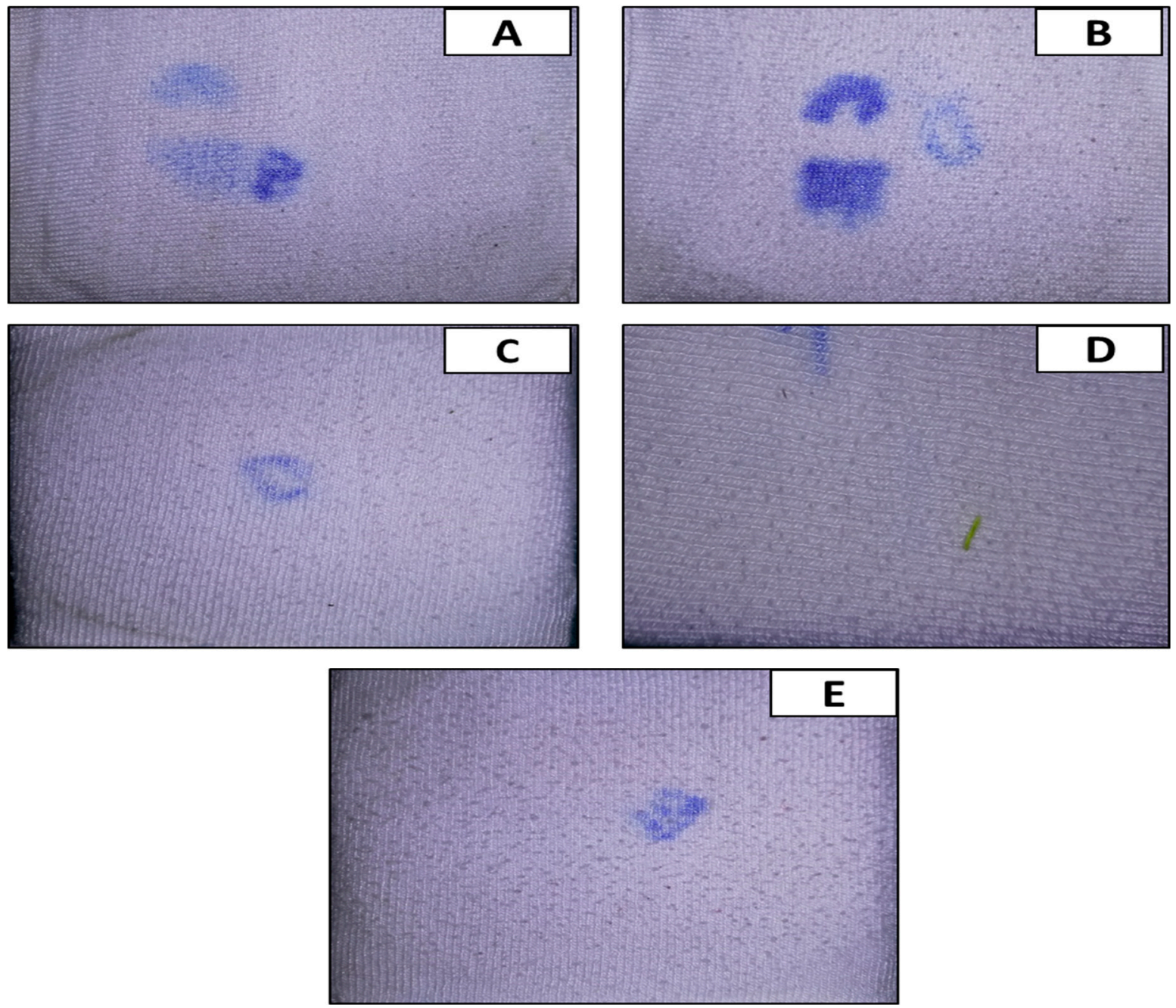

Figure 3. Picture of samples after finishing treatments (A) grey fabric, (B) simple wash, (C) normal finish, (D) silicon finish, and (E) wicking finish. 


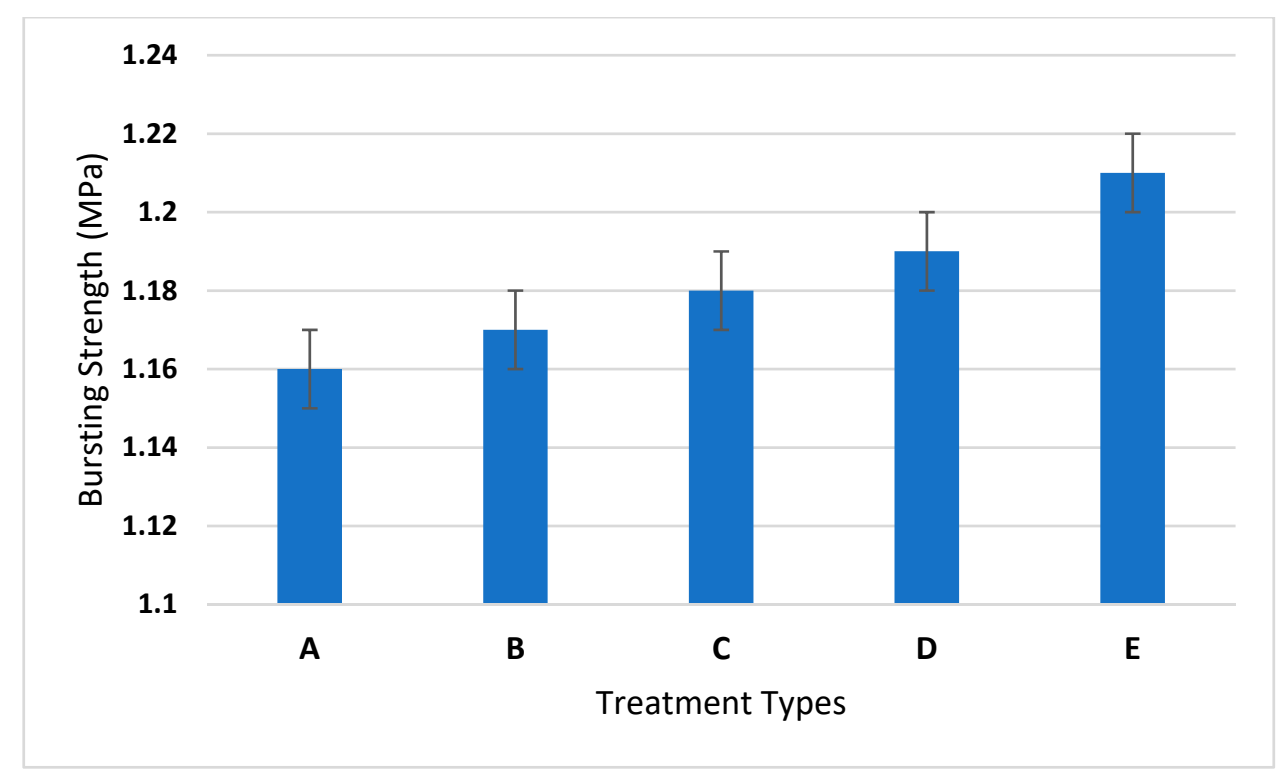

Figure 4. Effect of different finishes treatments on bursting strength (A) grey fabric, (B) simple wash, (C) normal finish, (D) silicon finish, and (E) wicking finish.

\subsection{Effect of Finishes Treatments on Wale-Wise and Course-Wise Vertical Wicking}

Wicking test results for wale-wise fabric samples revealed that the treatment of fabric with recipe $B$ shows an increase in wicking speed from 0.4 to $5 \mathrm{~mm} / \mathrm{min}$. Recipe $C$ treatment shows an increase of wicking speed from 0.4 and $5.5 \mathrm{~mm} / \mathrm{min}$. When the fabric was treated with recipe D wicking rate decrease from 0.4 to $0.33 \mathrm{~mm} / \mathrm{min}$. Recipe $\mathrm{E}$ increase from 0.4 to $5.1 \mathrm{~mm} / \mathrm{min}$. Thus, overall, treatments B, C, and E showed 12.50, 8, and 13.75-time increases in wicking speed, respectively. However, treatment $\mathrm{D}$ shows five-times decrease. In all treatments, wicking speed showed a significant increase as shown in Figure 5. Treatment $\mathrm{E}$ showed the highest increase in wicking speed, treatment $\mathrm{B}$ showed a medium increase in wicking speed, while treatment $C$ showed the least improvement in wicking speed. However, treatment D showed a slight decrease in the wicking rate. Because such finishes increased the hydrophilicity of the fabric except for silicon finishes which increase hydrophobicity. The hydrophilicity and hydrophobicity of the fabric directly affect the capillary action hence a significant increase has been observed in the vertical wicking rate by all treatment except treatment $\mathrm{D}$ (silicon wash) which reduce hydrophilicity and cause to deteriorate wicking rate [31].

Wicking test results for course-wise samples revealed that treatments B, C, and E show 10, 6.8, and 10.5 times increase in vertical wicking speed respectively. However, treatment D showed a 7.5-times decrease in the wicking rate. In all treatments, wicking speed showed a significant increase, except for treatment D, as shown in Figure 5 and Table 6. However, treatment E showed the highest increase in wicking speed. Treatment $C$ showed the least improvement in wicking speed while treatment $D$ showed a significant decrease. It has also been observed that the vertical wicking rate, of course, wise samples has been observed higher than that of the wale-wise samples because course density of the knitted fabric was mostly higher than wales density as shown in Table 1. Higher course density means lower distance amongst course-wise stitches which boost capillary action. On the other hand, lower wale density means more porosity and porosity prevent capillary action that is why the course-wise wicking rate is higher than the wale-wise wicking rate [32]. 
Table 6. Effect of finishes treatments on wale-wise and course-wise vertical wicking rate $\left(\mathrm{mm} \cdot \mathrm{min}^{-1}\right)$.

\begin{tabular}{ccccc}
\hline Treatment Type & $\begin{array}{c}\text { Wale-Wise } \\
\text { Wicking Rate } \\
\left(\mathbf{m m} \cdot \mathbf{m i n}^{-\mathbf{1}}\right)\end{array}$ & SD $( \pm)$ & $\begin{array}{c}\text { Course-Wise } \\
\text { Wicking Rate } \\
\left(\mathbf{m m} \cdot \mathbf{m i n}^{-\mathbf{1}}\right)\end{array}$ & SD $( \pm$ ) \\
\hline A & 0.4 & 0.09 & 0.6 & 0.11 \\
B & 5 & 0.17 & 6.2 & 0.21 \\
C & 3.2 & 0.13 & 4.1 & 0.18 \\
D & 0.33 & 0.21 & 0.52 & 0.25 \\
E & 5.5 & 0.24 & 6.3 & 0.20 \\
\hline
\end{tabular}

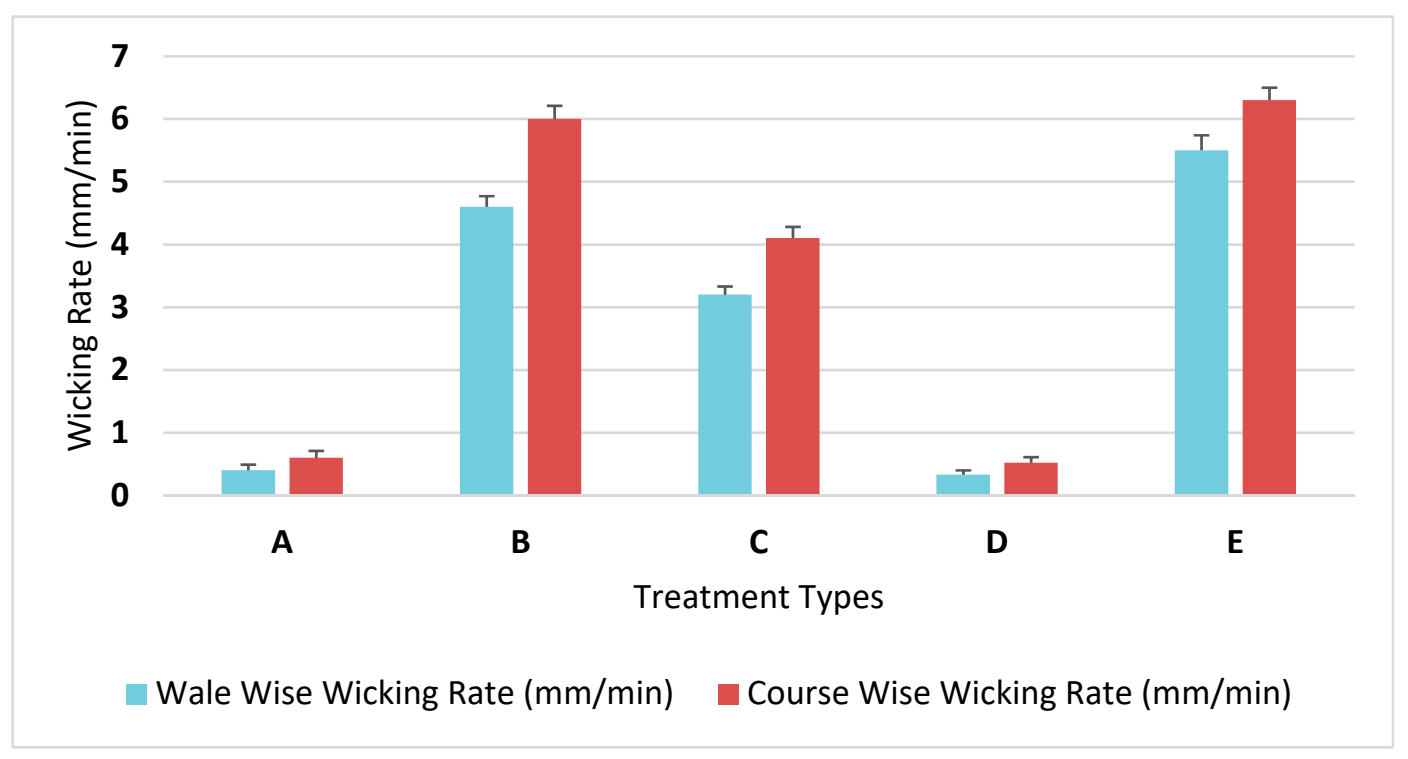

Figure 5. Effect of finishes treatments on wale-wise and course-wise vertical wicking behavior of $100 \%$ polyester weft-knitted jersey fabric. (A) Grey fabric, (B) simple wash, (C) normal finish, (D) silicon finish, and (E) wicking finish.

\section{Conclusions}

This study revealed the effect of washing, silicon softeners, and wicking finishes on the mass per unit area, pilling behavior, bursting strength, and wicking behavior of the polyester weft-knitted jersey fabric. Results revealed that mass per unit area (GSM) of the fabric increases by all treatments. However, treatment $\mathrm{E}$ showed the highest increase of $8.5 \%$ and treatments $\mathrm{B}$ and $\mathrm{C}$ showed the lowest increase in GSM of up to 5\%. Pilling showed deterioration by all treatments, however, treatments $B$ and $E$ showed the highest deterioration of 1 grade and $C$ and $D$ show the lowest deterioration by half a grade. Bursting strength of the fabric showed increases by all treatments, however, treatment $\mathrm{E}$ showed the highest increase of $4.2 \%$ and B showed the least increase of just $1.8 \%$. Vertical Wicking of the fabric showed significant improvement by all finishes in both wale-wise and course-wise except for treatment $\mathrm{D}$ which showed a significant reduction in the wicking rate. Treatment $\mathrm{E}$ showed the highest increase in wicking rate 13.75 times in the wale-wise direction and 10.5 times in the course-wise direction as compared to the grey fabric while treatment $C$ showed the lowest increase in wicking rate of 8 times in the wale-wise and 4 times in course-wise as compared to the grey fabric.

Author Contributions: Conceptualization, I.S.K.; data curation, M.Q.K., N.B., and Z.J.; formal analysis, I.S.K., M.Q.K., and A.R.; investigation, T.H., A.S., and N.H.; methodology, T.H.; project administration, M.Q.K. and I.S.K.; supervision, M.Q.K. and I.S.K.; visualization, T.H., M.Q.K., N.B., and Z.J.; writing-original draft, T.H.; writing-review \& editing, A.S., A.R., N.H., N.B., and Z.J. All authors have read and agreed to the published version of the manuscript.

Funding: This research received no external funding. 
Acknowledgments: Authors are thankful to Masood Textile Mill Faisalabad Pakistan for their support in this study.

Conflicts of Interest: The authors declare no conflicts of interest.

\section{References}

1. Chiweshe, A.; Crews, P.C. Influence of household fabric softeners and laundry enzymes on pilling and breaking strength. Text. Chem. Colorist Am. Dyest. Report. 2000, 32, 41-47.

2. Busilienè, G.; Lekeckas, K.; Urbelis, V. Pilling resistance of knitted fabrics. Mater. Sci. 2011, 17, $297-301$. [CrossRef]

3. Ukponmwan, A.M.J. Pilling. Text. Prog. 2009, 28, 1-57. [CrossRef]

4. Ukponmwan, J.O.; Mukhopadhyay, A.; Chatterjee, K.N. Pilling. Text. Prog. 1998, 28, 1-57. [CrossRef]

5. Cooke, W.D. Pilling attrition and fatigue. Text. Res. J. 1985, 55, 409-414. [CrossRef]

6. Kim, S.; Park, C.K. Evaluation of fabric pilling using hybrid imaging methods. Fibers Polym. 2006, 7, 57. [CrossRef]

7. Alston, P.V. Effect of Yarn Spinning System on Pill Resistance of Polyester/Cotton Knit Fabrics. Text. Res. J. 1992, 62, 105-108. [CrossRef]

8. Srinivasan, J.; Ramakrishnan, G.; Mukhopadhyay, S.; Manoharan, S. A study of knitted fabrics from polyester microdenier fibres. J. Text. Inst. 2007, 98, 31-35. [CrossRef]

9. Tadesse, M.G.; Nagy, L.; Nierstrasz, V.; Loghin, C.; Chen, Y.; Wang, L. Low-Stress Mechanical property study of various functional fabrics for tactile property evaluation. Materials 2018, 11, 2466. [CrossRef]

10. Milind, M.; Pant, S. Effect of resin finishes on pilling and dimensional stability of knitted fabrics. Man Made Text. India 2014, 42, 9-12.

11. Doustaneh, A.H.; Mahmoudian, S.; Mohammadian, M.; Jahangir, A. The effects of weave structure and yarn fiber specification on pilling of woven fabrics. World Appl. Sci. J. 2013, 24, 503-506.

12. Tusief, M.Q.; Mahmood, N.; Amin, N.; Saleem, M. Fabric tensile strength as affected by different anti pilling agents at various concentration and pH levels. J. Chem. Soc. Pak. 2013, 35, 238-242.

13. Akter Smriti, S.; Azharul Islam, M. An exploration on pilling attitudes of cotton polyester blended single jersey knit fabric after mechanical singeing. Sci. Innov. 2015, 3, 18-21. [CrossRef]

14. Brand, R.H.; Bohmfalk, B.M. A mathematical model of pilling mechanisms 1. Text. Res. J. 1967, 37, 467-476. [CrossRef]

15. Akter, N.; Akter, N. Effects of combed and carded yarn on weft knitted finished fabric quality. Int. J. Eng. Technol. IJET-IJENS 2011, 11, 93-97.

16. Hussain, T.; Safdar, F.; Nazir, A.; Iqbal, K. Optimizing the shrinkage and bursting strength of knitted fabrics after resin finishing. J. Chem. Soc. Pak. 2013, 35, 1452-1456.

17. Chowdhary, U.; Adnan, M.M.; Cheng, C. Bursting strength and extension for jersey, interlock and pique knits. Trends Text. Eng. Technol. 2018, 1, 19-27. [CrossRef]

18. Demiryürek, O.; Uysaltürk, D. Investigation on bursting strength and pilling properties of viloft/polyester blended knitted fabrics. J. Text. Eng. 2016, 23, 104-111.

19. Özbayrak, N.; Kavuşturan, Y. The effects of inlay yarn amount and yarn count on extensibility and bursting strength of compression stockings. J. Text. Apparel/Tekst. Konfeksiyon 2009, 19, 102-107.

20. Gong, R.H.; Bhatia, A. Effects of softeners on mechanical properties of cotton fabric. Res. J. Text. Appar. 2009, 13, 45-50. [CrossRef]

21. Khandaker, S.; Bhuiyan, M.A.; Hannan, M.A.; Al Faruque, M.A.; Azim, A.Y.M.; Rouf, M.A. Scope of polyester cotton blended single jersey knit fabric finishing without heat setting. Int. J. Sci. Eng. Technol. 2014, 3, 725-729.

22. Saricam, C. Absorption, wicking and drying characteristics of compression garments. J. Eng. Fibers Fabr. 2015, 10, 146-154. [CrossRef]

23. Gocek, İ.; Duru, S.C. Investigating the effects of wicking and antibacterial finishing treatments on some comfort characteristics of Meryl skinlife for seamless activewear/sportswear. J. Eng. Fibers Fabr. 2019, 14, 1558925019852790. [CrossRef]

24. Dong, Y.; Thomas, N.L.; Lu, X. Electrospun dual-layer mats with covalently bonded ZnO nanoparticles for moisture wicking and antibacterial textiles. Mater. Des. 2017, 134, 54-63. [CrossRef] 
25. ASTM D3776 Standard Test Methods for Mass Per Unit Area (Weight) of Fabric; ATSM: West Conshohocken, PA, USA, 2011.

26. ASTM D3512 Standard Test Method for Pilling Resistance and Other Related Surface Changes of Textile Fabrics: Random Tumble Pilling Tester Method; ASTM: West Conshohocken, PA, USA, 2005.

27. ASTM D3786/D3786M-13 Standard Test Method for Bursting Strength of Textile Fabrics-Diaphragm Bursting Strength Tester Method, D 3786/D3786 M-13; ASTM: West Conshohocken, PA, USA, 2013.

28. Kalkanci, M.; Sinecen, M.; Kurumer, G. Prediction of dimensional change in finished fabric through artificial neural networks. Tekst. Konfeksiyon 2018, 28, 43-51.

29. Harper, R.J., Jr.; Ruppenicker, G., Jr.; Donaldson, D. Cotton blend fabrics from polyester core yarns. Text. Res. J. 1986, 56, 80-86. [CrossRef]

30. Sharma, I.C.; Mukhopadhyay, D.; Agarwal, B.R. Feasibility of single jersey fabric from open-end spun blended yarn. Text. Res. J. 1986, 56, 249-253. [CrossRef]

31. Chowdhury, M.A. Improvement of moisture management of polyester fabric using moisture management chemical. Int. J. Eng. Technol. Manag. Appl. Sci. 2018, 5, 70-78.

32. Cloud, R.M.; Cao, W.; Song, G. Functional finishes to improve the comfort and protection of apparel. In Advances in the Dyeing and Finishing of Technical Textiles; Elsevier: Amsterdam, The Netherlands, 2013; pp. 258-279.

(C) 2020 by the authors. Licensee MDPI, Basel, Switzerland. This article is an open access article distributed under the terms and conditions of the Creative Commons Attribution (CC BY) license (http://creativecommons.org/licenses/by/4.0/). 